\title{
The macroscopic quantum state of ion channels: A carrier of neural information
}

\author{
Bo Song $^{1^{*}}$ and Lei Jiang ${ }^{1,2,3^{*}}$
}

\begin{abstract}
Neuroscience has been extensively developed for more than seventy years; however, there still remains lack of breakthrough understanding in the neural information field. A quantum coherent state of ion channels is recently proposed in neural system as an information carrier, but its physical expression is still not fixed. Here, employing a simple $\mathrm{K}^{+}$ channel as a typical instance, we theoretically build a conceptual model for the quantum state of a neural ion channel and demonstrate the macroscopic coherence state of multiple ion channels. The underlying mechanism is revealed to the mid-infrared photons released by ion oscillation in one ion channel together with their resonant and coherent coupling with the oscillations in other channels. An additional environment field (e.g., brain wave) may regulate the coherence, potentially relating to the human consciousness. Clearly, there exist the channels of other ions in a neural system, which also potentially form a macroscopic coherence state of themselves, with the mechanism identical to that of $\mathrm{K}^{+}$channels. An environment field can be expected to further regulate the quantum states of various ionic channels, leading to a total macroscopic coherence state of these channels, which is like the conductor in a symphony to achieve the harmony in music. These findings are expected to provide a promising viewpoint for neuroscience, as well as to improve treatments of the diseases and health problems related to neural system.
\end{abstract}

Keywords: macroscopic coherence state, biological ion channels, neural information

\section{INTRODUCTION}

Neuroscience has been extensively developed since the primitive voltage clamp in 1949 [1-5]; however, there still remains lack of breakthrough understanding in the neural information field. The related research is based on the
Hodgkin-Huxley $(\mathrm{HH})$ theory, where the generation of action potential (AP) is accompanied with the diffusion of ions driven by entropy along an axon, showing a slow propagation velocity $\left(0.2-120 \mathrm{~m} \mathrm{~s}^{-1}\right)$ [6,7]. Meanwhile, the ion diffusion of AP conduction will need an amount of energy, limited by the laws of thermodynamics. The properties above cannot agree with the rapid transmission of neural information with a huge amount of data and an ultralow energy consumption $(\sim 60 \mathrm{~W})$, suggesting that AP may not have a significant correlation with actually instantaneous responses of neural system, especially of the brain [8]. Besides the classical HH theory, the quantum bio-information approaches have also been proposed recently, including the neural qubits based on quantum entanglement of Posner molecules [9] and the quantum radical-pair model for avian magneto-reception [10]. But up to now, the acceptable quantum state of neural information and its carrier are still undetermined.

Recently, inspired by the high-flux transport of ordered ions across a biological channel and the shock generation of electric eels [11-14], we have proposed a quantumconfined ion superfluid (QISF) in ionic channel as a quantum state of bio-information, followed by a coherence state of the channels which may act as a neural information carrier $[8,15]$. The high flux is attributed to the one-dimensional (1D) collective motion of ions confined in an ionic channel. This manner is specifically caused by both the confinement space of channel comparable to the Debye distance of ion diffusion and the equivalent attraction between the ions (such as $\mathrm{K}^{+}$) induced by negative charges in the inner surface of channel [16]. On the other hand, within the millisecond magnitude, millions of electrically active cells of an electric eel can generate a shock over $600 \mathrm{~V}$, implying a coherence of

\footnotetext{
${ }^{1}$ School of Optical-Electrical Computer Engineering, University of Shanghai for Science and Technology, Shanghai 200093, China

${ }^{2}$ Key Laboratory of Bio-inspired Materials and Interfacial Science, Technical Institute of Physics and Chemistry, Chinese Academy of Sciences, Beijing 100190, China

${ }^{3}$ School of Future Technology, University of Chinese Academy of Sciences, Beijing 100049, China

* Corresponding authors (emails: bsong@usst.edu.cn (Song B); jianglei@iccas.ac.cn (Jiang L))
} 
ionic channels (i.e., a resonant ion wave), rather than an ionic diffusion process [12-14]. All of these facts suggest a quantum coherent state of ion channels existing in a neural system, which may act as the carrier of neural information. However, the expression of the coherent state is not fixed.

In current work, we try to theoretically build a conceptual model for the quantum state of an ion channel and the coherent state of multiple ion channels. Amounts of different channels exist in the neural system, including the $\mathrm{Na}^{+}, \mathrm{K}^{+}$and $\mathrm{Ca}^{2+}$ channels $[11,17-22]$. There are at least five types of $\mathrm{K}^{+}$channels [20]; the simplest one (i.e., MthK [22]) is employed to demonstrate the concept. The mechanism under the channel coherence is revealed to both the mid-infrared (MIR) photons released by ion oscillation in one channel and their resonant coupling with the ion oscillations in other channels. Moreover, additional environment fields including brain waves may regulate the coherent state, which potentially relates to the consciousness of human.

\section{AN EXPRESSION OF QUANTUM STATE FOR THE CONFINED IONS IN A $\mathrm{K}^{+}$ CHANNEL}

As a typical example, the MthK channel of $\mathrm{K}^{+}$selectivity is shown in Fig. 1a. The tube-like filter is the key region for the ion selection, whose two terminals are connected by a small cavity and a large one, respectively. The filter region has a length of $\sim 1.2 \mathrm{~nm}$ and a diameter of $\sim 0.25 \mathrm{~nm}$ [22], showing its diameter consistent with that of $\mathrm{K}^{+}$ion $(0.27 \mathrm{~nm})$ [23]. The function of selecting $\mathrm{K}^{+}$ ions is performed by four rings on the inner surface of filter, where each ring consists of four carbonyl groups and each oxygen atom of carbonyl group has a charge of -0.5 e approximately. $\mathrm{K}^{+}$ions are orderly confined in the ion-selective filter with almost no change [20,24]. The above information provides a chance for us to build a model in the presence of confinement potential and membrane electric field (Fig. 1b) [16]. Let us firstly consider a diameter-fixed channel for the motion of $\mathrm{K}^{+}$ ions in the filter region, with the diameter $D=0.3 \mathrm{~nm}$ and length $L=1.2 \mathrm{~nm}$. Four rings were added to its inner surface, and each ring contained four charged particles with -0.5 e corresponding to the negatively-charged oxygen atoms above. An attractive potential thus forms for the $\mathrm{K}^{+}$ions outside (Fig. $1 \mathrm{~b}$ middle left), and then three $\mathrm{K}^{+}$ions can be confined in the tube, presenting a behavior of linear lattice. Hence, we built a 1D lattice model for these ions (Fig. 1b right), with a pseudo-anion with charge of -2.0 e simplified from the negatively

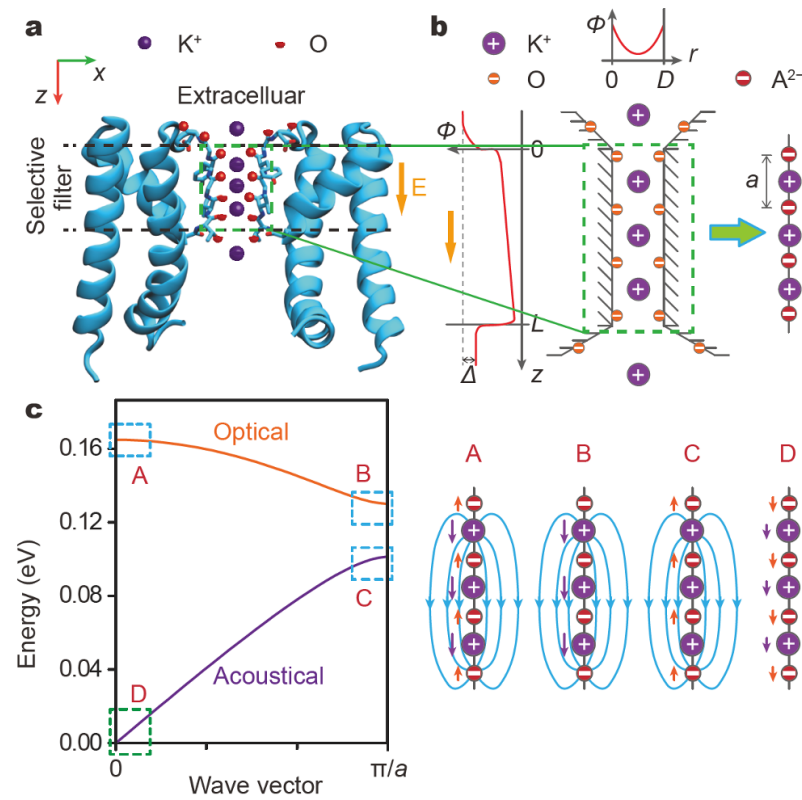

Figure 1 1D lattice model of $\mathrm{K}^{+}$ions confined in a $\mathrm{K}^{+}$channel and the emitted EM wave. (a) A typical protein structure of $\mathrm{K}^{+}$selectivity channel with an electric field $E$ induced by the membrane potential. (b) The modelling of $\mathrm{K}^{+}$channel. $\mathrm{K}^{+}$ions are confined in a $\mathrm{K}^{+}$channel by the potentials along the axis (left) and radial (upper) directions. The orange circle indicates the negatively charged oxygen atom for the selectivity. Right: 1D lattice model for the ions confined in the ion-selective filter. The dark red circle indicates the pseudo-anion introduced from four negatively charged oxygen atoms with an identical $z$ value. (c) The properties of $\mathrm{K}^{+}$ions confined in ion-selective filter. Left: the dispersion with optical and acoustical lattice waves; right: the typical vibration modes and their emitted EM waves (weak blue curves).

charged ring. The length $a$ of a unit cell was set as $0.4 \mathrm{~nm}$ according to the MthK structure.

Based on the 1D lattice model, the quantum state of confined ions in a $\mathrm{K}^{+}$channel is explored. Firstly, we obtained the dispersion relation with optical $\left(\omega_{\mathrm{O}}\right)$ and acoustical $\left(\omega_{\mathrm{A}}\right)$ branches [25],

$$
\begin{aligned}
& \omega_{\mathrm{O}}^{2}=\frac{C_{1}+C_{2}}{2 m M}\left\{\Xi+\sqrt{\Xi^{2}-\frac{16 m M C_{1} C_{2}}{\left(C_{1}+C_{2}\right)^{2}} \sin ^{2}(k a / 2)}\right\}, \\
& \omega_{\mathrm{A}}^{2}=\frac{C_{1}+C_{2}}{2 m M}\left\{\Xi-\sqrt{\Xi^{2}-\frac{16 m M C_{1} C_{2}}{\left(C_{1}+C_{2}\right)^{2}} \sin ^{2}(k a / 2)}\right\},
\end{aligned}
$$

where $\Xi=M+m, M$ and $m$ indicate the masses of pseudo-anion (i.e., four negatively charged oxygen atoms) and $\mathrm{K}^{+}$ion, $C_{1}$ and $C_{2}$ mean the coupling constant of force between the nearest neighboring particles, while $k$ denotes the wave vector. As the results of dispersion shown in Fig. 1c, there are four typical modes of lattice waves. Namely, the long wavelength $(k \rightarrow 0$, the wavelength $\lambda \rightarrow \infty)$ and short wavelength limits $(k \rightarrow \pi / a, \lambda \rightarrow$ 
$2 a$ ) of optical and acoustical branches, respectively, labeled as the modes A, B, C and D, respectively (see the details in Fig. 1c right). The relative motion of pseudoanions and $\mathrm{K}^{+}$ions in the modes $\mathrm{A}, \mathrm{B}$ and $\mathrm{C}$ corresponds to a multiple oscillation and thus can emit an electromagnetic (EM) wave, which will not occur in the mode $\mathrm{D}$ due to no relative motion of the negative and positive ions.

To determine the frequency and wavelength of lattice waves in the $\mathrm{K}^{+}$channel, we have performed ab initio calculations on a MthK protein (PDB IDs: 3ldc). The B3LYP $[26,27]$ exchange correlation functional and 6-31 $+\mathrm{G}(\mathrm{d})$ basis set were employed in the density functional theory (DFT) calculations. The geometry optimization was performed first to obtain the stable conformations of molecules and the lowest energy conformation was applied for calculating results. All calculations were performed by the Gaussian 16 software [28]. The resulting coupling constant $C_{0}$ along the channel-axis direction was $3.33 \mathrm{nN} \AA^{-1}$ of $\mathrm{K}^{+}$ion and its neighboring $\mathrm{O}$ atoms without any membrane potential. The potential will introduce a perturbation $\delta\left(<C_{0}\right)$ to the coupling, approximately leading to $C_{1}=C_{0}+\delta$ and $C_{2}=C_{0}-\delta$. Through the dispersion relation, the acoustical and optical branches were determined in the ranges of $0-0.10 \mathrm{eV}$ and $0.13-0.17 \mathrm{eV}$, respectively, corresponding to $0-$ $24 \mathrm{THz}$ and $29-41 \mathrm{THz}$. Note that the frequencies of optical branch just fall in the MIR region. The wave function at the short wavelength limit $k \rightarrow \pi / a$ of optical branch was written in a simple form of standing wave $u_{s}(t)=A \cos (k \cdot s a) \exp (-i \omega t+\varphi)$, where $u, A, \varphi$ and $s$ mean the displacement of $\mathrm{K}^{+}$ion, the maximum amplitude and initial phase of vibration, and the index of lattice site, respectively. In principle, a lattice wave can be quantized to a phonon, and the corresponding energy is [25]

$E_{N}(\omega)=(N+1 / 2) \hbar \omega$,

where $N$ indicates the number of phonons with the energy $\hbar \omega$, while the frequency $\omega$ is given by the dispersion. For the mode of the short wavelength limited in the optical branch, the quantum wave function is simply expressed as

$\psi(z, t)=A_{N} \cos \left(k_{z} \cdot z\right) \exp (-i \omega t+\varphi)$,

with $\left|A_{N}\right|^{2} \propto N, \omega=\omega_{\mathrm{O}} \approx \sqrt{2 C_{0} / m}$ and $k_{z}=k=2 \pi / \lambda$ according to the 1D lattice model. For other modes, we had $\omega=\omega_{\mathrm{O} / \mathrm{A}}(k)$. Introducing a periodical condition to our applied channel of $L=3 a$, we had a limitation of $k=$ $K \cdot 2 \pi / 6 a$ with $K=1,2$ and 3 to the confined phonons [29], leading to $\lambda=6 a / K(K=3$ for the short wavelength limit $\lambda=2 a$ ), and $L=K \cdot \lambda / 2$ for the confinement. The $L \sim$
$1.2 \mathrm{~nm}$ and $a \sim 0.4 \mathrm{~nm}$ of MthK protein yield the wavelengths of 2.4, 1.2, and $0.8 \mathrm{~nm}$ for the confined states with $K=1,2$ and 3 , respectively. The corresponding frequency is readout in Fig. 1c with the help of the wave vector $k=$ $K \pi / 3 a$. We thus can conclude that the space confinement of channel and the negatively charged oxygen atoms on the inner surface of channel orderly align the $\mathrm{K}^{+}$ions to a 1D lattice, leading to the quantum state of ion vibration. Oscillation (especially the optical mode in $29-41 \mathrm{THz}$ ) of the confined $\mathrm{K}^{+}$ions can emit an EM wave with the frequency identical to that of ion oscillation (Fig. 1c right).

\section{A MACROSCOPIC QUANTUM STATE OF MULTIPLE ION CHANNELS}

Generally for the formation of a macroscopic quantum state, two conditions are necessary: (1) all the elements oscillate with a same frequency, and (2) the oscillation phases come to be homogeneous. The laser is a typical example of macroscopic quantum states [30]. For laser emission, a large amount of photons $h v_{2}$ with an identical frequency are released due to the population inversion of gain medium pumped by light $h v_{1}$ (Fig. 2a left). The released photons then travel between two mirrors of resonant cavity which acts as an environment regulator. The distance $D$ of mirrors is set to an integral multiple of half wavelength of the photons, and then the periodicity of photon traveling between them is related to the environment frequency for regulating the photon phases to an identical value of coherence (Fig. 2a right). Another typical example of macroscopic coherence phenomenon is visually shown by $32(8 \times 4)$ metronomes with a frequency of $0.25 \mathrm{~Hz}$ (Fig. 2b) [31]. The metronomes are placed on a hung plane, while their initial phases $\varphi_{i}(i=1$ to 32) are set randomly. The resonance wave formed by the oscillations of most metronomes drives the plane to oscillate with a specific frequency $\omega_{\mathrm{p}}$, which in turn regulates the random phases $\varphi_{i}$ to a same value $\varphi_{0}$, leading to a coherent state of all metronomes. Therefore, the formation of macroscopic coherence state can be attributed to the regulation effect of environment oscillation induced by frequency-identical elements on the phases of these elements.

We suggest a macroscopic coherence state of ion channels in neural system, which may relate to a fully clear consciousness, and then the decoherence of this state relates to the fully confusing one (Fig. 3a). A coherent state formation of neural ion channels is similar to the case of metronomes. A kind of ion channels shares a same structure of key section, and thus has an identical 


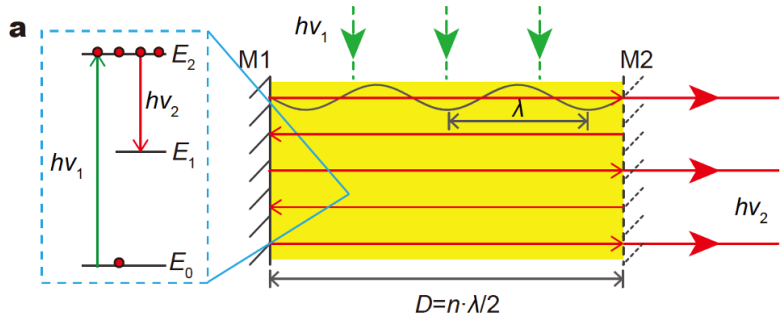

b
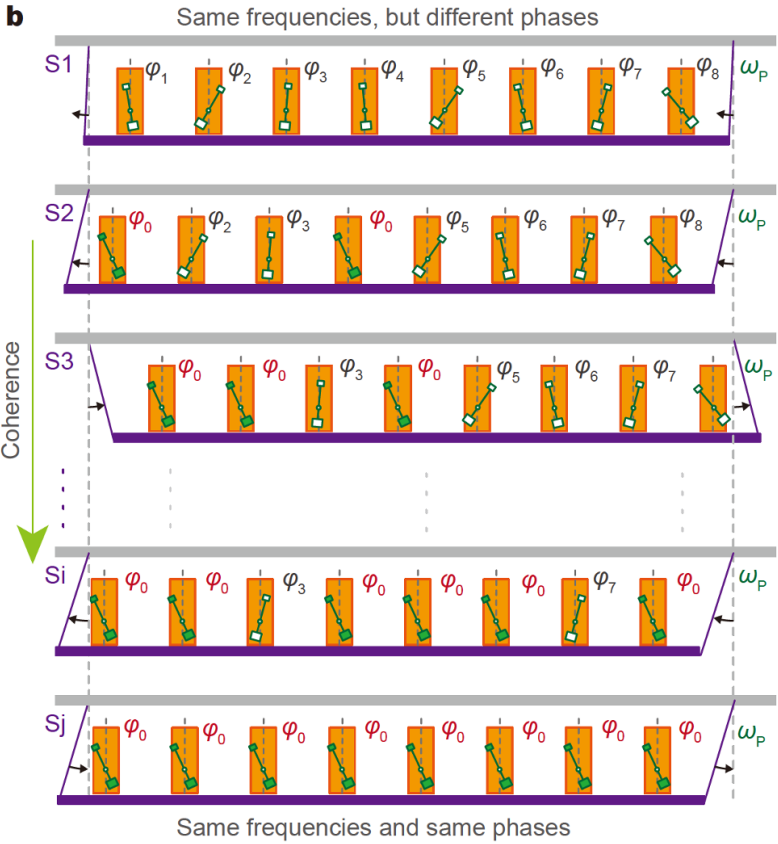

Figure 2 Typical macroscopic coherence states forming under specific conditions. (a) Coherence of photons in a laser. An optical resonant cavity formed by fully (M1) and partially (M2) reflective mirrors regulates the photons released by a gain medium (yellow) to a coherent state. (b) Coherence process of frequency-identical metronomes on a hung plane. The label $\omega_{\mathrm{p}}$ indicates the frequency of plane vibration.

frequency of confined ion vibration, providing a basis of coherence. The average distance of ion channels is $\sim 1 \mu \mathrm{m}$ $[32,33]$, clearly less than the EM wavelength of $7-10 \mu \mathrm{m}$ (see the calculation results in the previous section) emitted by the ion vibration in channel. Adenosine triphosphate (ATP) can release photons with a frequency of $\sim 34 \mathrm{THz}$ (wavelength $\sim 8.4 \mu \mathrm{m}$ ) for resonantly driving a bioprocess [34], which just falls in the optical branch (29$41 \mathrm{THz}$, see the previous section) of confined ion vibration in channel. ATP as a biological energy currency can thus always provide energy for the ion channels to maintain their dynamical coherence. Most of multipole oscillations in ion channels potentially form a resonance wave (such as brain waves [35]) as the environment regulator of coherence. As for the example of MthK channel, the multipole oscillation of confined ions can emit EM waves with a frequency identical to that of the ionic vibration (Fig. 1c right). The waves released by different $\mathrm{K}^{+}$channels have a same frequency, but random phases (Fig. 3a top). The environment regulator (e.g., the $\delta$ brain wave $\omega_{\mathrm{B}}<4 \mathrm{~Hz}$ ) will drive these phases to an identical value, leading to a coherence state of ion channels (Fig. 3a bottom). Meanwhile, the environment wave $\omega_{\mathrm{B}}$ is replaced by another one $\omega_{\mathrm{B}}^{*}$ (e.g., the $\beta$ or $\gamma$ brain wave of $>12 \mathrm{~Hz}$ ).

The above process can be physically expressed in a spectral space. Due to the consistency in frequencies and the matching in momenta, the EM wave released from one channel can be resonantly absorbed by the $\mathrm{K}^{+}$ions in the other channels with a high efficiency and thus regulate their oscillation phases, inducing a vibration coherence of confined ions in the channels step by step. After quantization, a quantum coherent state of the $\mathrm{K}^{+}$ channels was obtained, which can be approximately expressed in a physically simplified form.

$$
\begin{aligned}
& \psi_{i}(z, t)=A_{N} \cos \left(k_{z} \cdot z\right) \exp \left(-i \omega t+\varphi_{i}\right), \\
& \boldsymbol{\Psi}_{\mathrm{EM}, i}(x, y ; t)= \boldsymbol{A}_{\mathrm{EM}, i} \cos \left(k_{\mathrm{EM}, x} \cdot x\right) \cos \left(k_{\mathrm{EM}, y} \cdot y\right) \\
& \cdot \exp \left(-i \omega t+\varphi_{i}\right), \\
& \boldsymbol{\Phi}_{\mathrm{env}}(x, y ; t)= \boldsymbol{A}_{\mathrm{env}} \cos \left(k_{\mathrm{env}, x} \cdot x\right) \cos \left(k_{\mathrm{env}, y} \cdot y\right) \\
& \cdot \exp \left(-i \omega_{\mathrm{env}}(t) \cdot t\right) .
\end{aligned}
$$

Equation (4a) indicates the multipole oscillation $\psi_{i}$ in the ion channel $i$ with a phase $\varphi_{i}$ (Fig. 3a top) along the axis direction of channel (i.e., the $z$ direction). Equation (4b) denotes the EM field $\Psi_{\mathrm{EM}}$ released by the oscillation in the channel $i$, which majorly propagates in the direction vertical to the channel axis. The $z$ component of wave vector $\boldsymbol{k}_{\mathrm{EM}}=\left(k_{\mathrm{EM}, x}, k_{\mathrm{EM}, y}, k_{\mathrm{EM}, z}\right)$ can thus be approximated to vanishing (i.e., $k_{\mathrm{EM}, z} \rightarrow 0$ ). The label $\boldsymbol{A}_{\mathrm{EM}, i} \perp$ $\boldsymbol{k}_{\mathrm{EM}}$ means the amplitude vector of EM field. A collective effect of these two kinds of fields will spontaneously induce an environment wave $\boldsymbol{\Phi}_{\text {env }}$ in Equation (4c). The induced wave propagates vertically to the channel axis, i.e., the wave vector $\boldsymbol{k}_{\mathrm{env}}=\left(k_{\mathrm{env}, x}, k_{\mathrm{env}, y}, 0\right)$, while its frequency $\omega_{\text {env }}$ depends on time and the wave amplitude vector $\boldsymbol{A}_{\mathrm{env}}=\left(0,0, A_{\mathrm{env}, z}\right)$ is along the channel axis. By the help of environment wave, resonant coupling of the photons (i.e., quantized EM wave) and ion oscillations will cause a coherence of these channels, i.e., all $\varphi_{i}=\varphi_{0}$ (Fig. 3a bottom). Indeed, the brain wave potentially corresponds to the environment wave caused by ion oscillations of neural channels.

From a macroscopic view of human body (Fig. 3b), coherent and decoherent states can be expected to form 

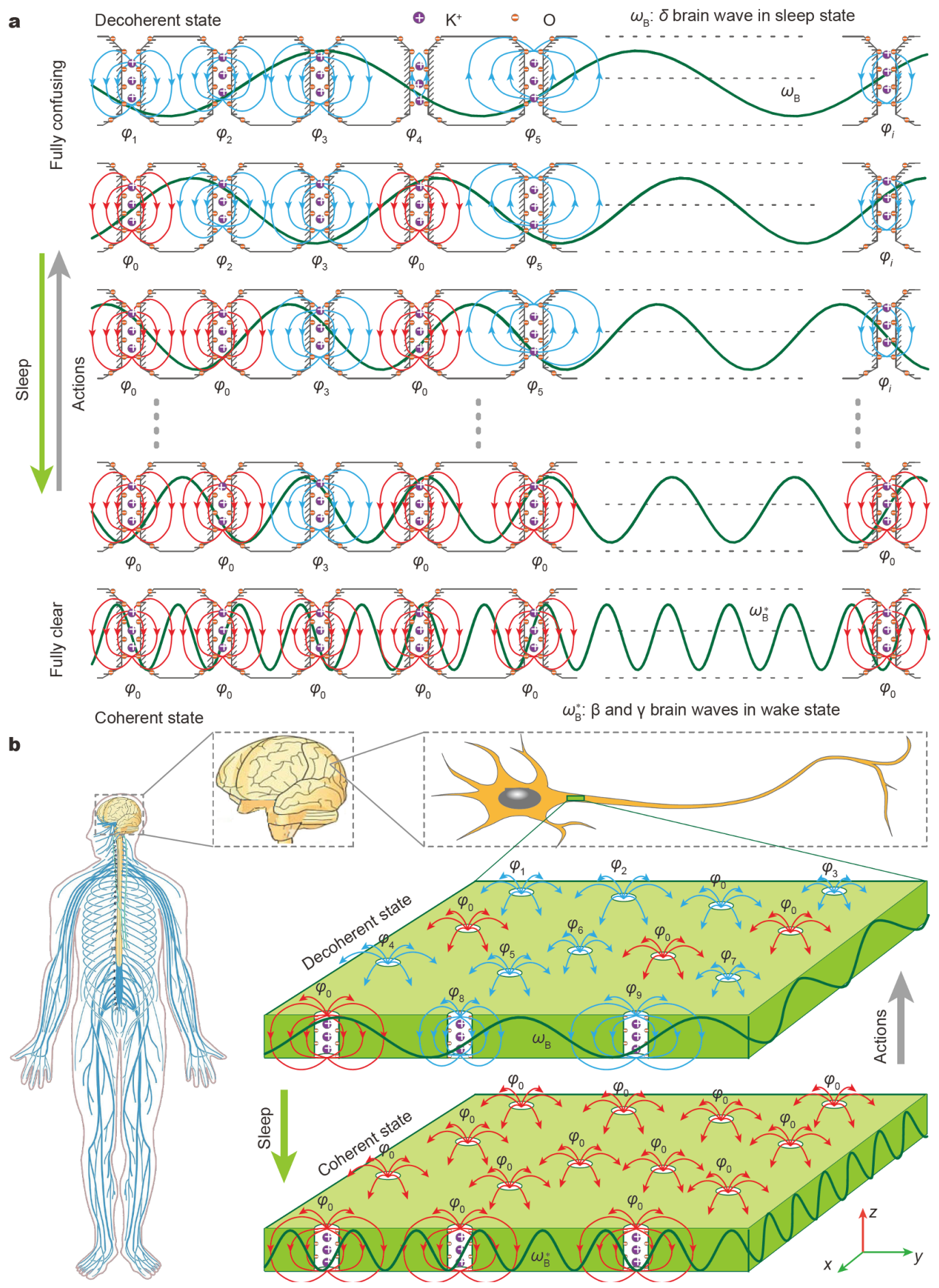

Figure 3 Macroscopic coherence state of $\mathrm{K}^{+}$channels resonantly coupled with the photons released by the ion oscillations in the channels and regulated by a brain wave. (a) Oscillation of $\mathrm{K}^{+}$ions confined in a $\mathrm{K}^{+}$channel can release MIR photons (the quantized EM waves) with a frequency identical to the oscillating one. The phases $\varphi_{i}$ of ion vibrations in the channels $i$ will come to be an identical value $\varphi_{0}$ by the resonant coupling with these photons, causing a quantum state of multiple $\mathrm{K}^{+}$channels in neuron. The blue and red curves represent the EM waves with random and identical phases, respectively. (b) A macroscopic coherence state in the brain. The green and gray arrows stand for the processes of coherence (sleep) and de-coherence (actions), respectively. The green region (upper right) indicates the fragment of neuron which has an amount of $\mathrm{K}^{+}$channels (lower right) with an inter distance $\sim 1 \mu \mathrm{m}$. 
in all the ion channels of neural system, which may correspond to the fully clear and fully confusing states of consciousness, respectively. In a fragment of neuron as shown in Fig. 3b upper right, the ions in different channels oscillate with a same frequency and random phases, and thus can emit the corresponding EM waves, which potentially relates to a decoherence state of brain. Regulated by the environment wave $\omega_{\mathrm{B}}$ in sleep state (e.g., $\delta$ brain wave), the oscillation phases of ion channels come to homogenous step by step, leading to a coherence state of them, while the wave $\omega_{\mathrm{B}}$ becomes a new wave $\omega_{\mathrm{B}}^{*}$ (e.g., $\beta$ or $\gamma$ brain wave) of fully clear consciousness. Usually, the coherence will be disturbed by amounts of various neural signals for controlling the activities in awake state, during which this coherent state comes to be decoherent in a time approximately of eight hours or more depending on the individual difference. After that, the consciousness becomes confusing, related to the decoherence state of brain. The sleep is then needed again for rebuilding the coherence state. Here, the $\mathrm{K}^{+}$channel as a typical instance is applied to demonstrate the physics. Clearly, the neural system has other ionic channels, which also potentially form a macroscopic coherence state of themselves, with the same mechanism as that of $\mathrm{K}^{+}$ channels. Then an additional field with a special frequency (e.g., the environmental field) can be expected to regulate the quantum states of various ionic channels, leading to a total macroscopic coherence state of these channels. Thus it could be the reason for that 6-8 h sleep is needed to make these channels coherent. This is similar to how an orchestra conductor (the additional environment field) unifies a large group of musicians with different instruments (the different ion channels with different frequencies) into music harmony (the total macroscopic coherence state). These states may correspond to the high efficiency, ultralow energy consumption and huge amount of data in the rapid transmission of neural information. Besides the nervous system of consciousness, the coherent state in autonomic nervous system may be also important for us. For example, a kind of spontaneous and persistent coherence state of $\mathrm{Ca}^{2+}$ channels potentially should exist in our heart, which controls the periodical twitch of myocardia, resulting in the beat of heart and then sustaining our lives. Consequently, the cardiac arrest may be caused by an emergency of decoherence in these $\mathrm{Ca}^{2+}$ channels (Personal communication of Wang J). Cardiopulmonary resuscitation can restart the heartbeat, in which the periodical compression with a frequency of $\sim 2 \mathrm{~Hz}$ may play the role of environment field in rebuilding the coherent state of these $\mathrm{Ca}^{2+}$ channels, causing the reboot of periodical myocardium twitch. The corresponding explorations should be addressed in future.

\section{CONCLUSION}

In summary, using the $\mathrm{K}^{+}$-selectivity channel as a typical example, we demonstrate a form of the quantum state of ion vibration confined in a neural ion channel as well as the coherent state of multiple ion channels. The mechanism is further revealed to that the multipole oscillation of confined ions in each channel can release photons in MIR region, which will be resonantly absorbed by the ion oscillations in the other channels and then affect them. The coupling of ion channels and their released photons would induce an environment wave, which in turn regulates the ion oscillations in the channels to a coherence state of them. In spite of the diversity of biological channels [17], $\mathrm{Na}^{+}$- and $\mathrm{K}^{+}$-selectivity channels play a key role in neural information transmission. Based on the identical physics, $\mathrm{Na}^{+}$-selectivity channels can be expected to form similar states to the results here (but with a different frequency), which would be explored in future. The environment field (e.g., brain waves) may play pivotal roles in orchestrating the macroscopic quantum states of various ionic channels. This kind of states may occur in the neural system, leading to a microscopic neuro coherence state that potentially acts as a carrier of our consciousness. Such states are reasonable for the high efficiency, high precision and ultralow energy consumption of neural information more than the ion diffusion of AP conduction. The findings would provide a promising viewpoint for neuroscience, and even to improve treatments of the diseases related to our neural system, especially the health problems related to sleep.

Received 20 January 2021; accepted 8 February 2021; published online 28 May 2021

1 Marmont G. Studies on the axon membrane. I. A new method. J Cell Comp Physiol, 1949, 34: 351-382

2 Neher E, Sakmann B. Single-channel currents recorded from membrane of denervated frog muscle fibres. Nature, 1976, 260 : 799-802

3 Duan X, Gao R, Xie $\mathrm{P}$, et al. Intracellular recordings of action potentials by an extracellular nanoscale field-effect transistor. Nat Nanotech, 2012, 7: 174-179

4 Williamson A, Ferro M, Leleux P, et al. Localized neuron stimulation with organic electrochemical transistors on delaminating depth probes. Adv Mater, 2015, 27: 4405-4410

5 Fu TM, Hong G, Zhou T, et al. Stable long-term chronic brain mapping at the single-neuron level. Nat Methods, 2016, 13: 875882

6 Hodgkin AL, Huxley AF. A quantitative description of membrane 
current and its application to conduction and excitation in nerve. J Physiol, 1952, 117: 500-544

7 Parker JL, Shariati NH, Karantonis DM. Electrically evoked compound action potential recording in peripheral nerves. Bioelectron Med, 2018, 1: 71-83

8 Zhang X, Jiang L. Quantum-confined ion superfluid in nerve signal transmission. Nano Res, 2019, 12: 1219-1221

9 Fisher MPA. Quantum cognition: The possibility of processing with nuclear spins in the brain. Ann Phys, 2015, 362: 593-602

10 Lambert N, Chen YN, Cheng YC, et al. Quantum biology. Nat Phys, 2013, 9: 10-18

11 Zhou Y, Morais-Cabral JH, Kaufman A, et al. Chemistry of ion coordination and hydration revealed by a $\mathrm{K}^{+}$channel-Fab complex at $2.0 \AA$ resolution. Nature, 2001, 414: 43-48

12 Ruiz-Manresa F, Grundfest H. Synaptic electrogenesis in eel electroplaques. J General Physiol, 1971, 57: 71-92

$13 \mathrm{Xu}$ J, Lavan DA. Designing artificial cells to harness the biological ion concentration gradient. Nat Nanotech, 2008, 3: 666-670

14 de Santana CD, Crampton WGR, Dillman CB, et al. Unexpected species diversity in electric eels with a description of the strongest living bioelectricity generator. Nat Commun, 2019, 10: 4000

15 Zhang X, Antonietti M, Jiang L. Bioinformation transformation: From ionics to quantum ionics. Sci China Mater, 2020, 63: 167171

16 Schoch RB, Han J, Renaud P. Transport phenomena in nanofluidics. Rev Mod Phys, 2008, 80: 839-883

17 Hille B. Ionic Channels of Excitable Membranes. $3^{\text {rd }}$ Ed. Sunderland: Sinauer Associates Inc., 2001

18 Zhang X, Ren W, DeCaen P, et al. Crystal structure of an orthologue of the NaChBac voltage-gated sodium channel. Nature, 2012, 486: $130-134$

19 Kratochvil HT, Carr JK, Matulef K, et al. Instantaneous ion configurations in the $\mathrm{K}^{+}$ion channel selectivity filter revealed by $2 \mathrm{D}$ IR spectroscopy. Science, 2016, 353: 1040-1044

20 Kopec W, Köpfer DA, Vickery ON, et al. Direct knock-on of desolvated ions governs strict ion selectivity in $\mathrm{K}^{+}$channels. Nat Chem, 2018, 10: 813-820

21 Doyle DA, Morais Cabral J, Pfuetzner RA, et al. The structure of the potassium channel: Molecular basis of $\mathrm{K}^{+}$conduction and selectivity. Science, 1998, 280: 69-77

22 Ye S, Li Y, Jiang Y. Novel insights into $\mathrm{K}^{+}$selectivity from highresolution structures of an open $\mathrm{K}^{+}$channel pore. Nat Struct Mol Biol, 2010, 17: 1019-1023

23 Shannon RD. Revised effective ionic radii and systematic studies of interatomic distances in halides and chalcogenides. Acta Cryst A, 1976, 32: 751-767

24 Holyoake J, Domene C, Bright JN, et al. KcsA closed and open: Modelling and simulation studies. Eur Biophys J, 2004, 33: 238246

25 Kittel C. Introduction to Solid State Physics. $8^{\text {th }}$ Ed. New York: John Wiley \& Sons, Inc., 2005

26 Becke AD. Density-functional thermochemistry. III. The role of exact exchange. J Chem Phys, 1993, 98: 5648-5652

27 Lee C, Yang W, Parr RG. Development of the Colle-Salvetti correlation-energy formula into a functional of the electron density. Phys Rev B, 1988, 37: 785-789

28 Frisch MJ, Trucks GW, Schlegel HB, et al. Gaussian 16. Revision B.01. Wallingford CT: Gaussian, Inc., 2016

29 Song B, Shu Y. Cell vibron polariton resonantly self-confined in the myelin sheath of nerve. Nano Res, 2020, 13: 38-44
30 Denker B, Shklovsky E. Handbook of Solid-State Lasers. Cambridge: Woodhead Publishing Limited, 2013

31 Ikeguchi T, Shimada Y. Analysis of synchronization of mechanical metronomes. In: Proceedings of the 5th International Conference on Applications in Nonlinear Dynamics. Maui, 2019. 141-152

32 Leterrier C, Potier J, Caillol G, et al. Nanoscale architecture of the axon initial segment reveals an organized and robust scaffold. Cell Rep, 2015, 13: 2781-2793

33 Xu K, Zhong G, Zhuang X. Actin, spectrin, and associated proteins form a periodic cytoskeletal structure in axons. Science, 2013, 339: 452-456

34 Li N, Peng D, Zhang X, et al. Demonstration of biophoton-driven DNA replication via gold nanoparticle-distance modulated yield oscillation. Nano Res, 2021, 14: 40-45

35 Abhang PA, Gawali BW, Mehrotra SC. Introduction to EEG- and Speech-Based Emotion Recognition. Amsterdam: Academic Press, 2016

Acknowledgements This work was supported by the National Key Research and Development Program of China (2018YFE0205501), the National Natural Science Foundation of China Project (21988102) and the National Supercomputer Center in Tianjin.

Author contributions Song B and Jiang L designed and performed the research, and wrote the paper together.

Conflict of interest The authors declare that they have no conflict of interest.

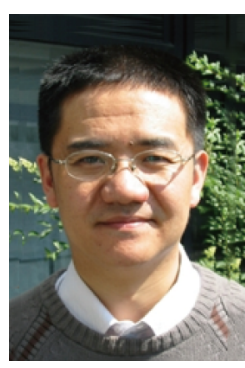

Bo Song is a professor at the University of Shanghai for Science and Technology (USST). He received his $\mathrm{PhD}$ from the Technical Institute of Physics and Chemistry (TIPC), Chinese Academy of Sciences. He worked as a postdoctoral fellow with Prof. Wenjian Liu at Peking University and then with Prof. Giovanni Cuniberti at Regensburg University and the Technische Universität Dresden. In 2008, he joined Shanghai Institute of Applied Physics, Chinese Academy of Sciences. In 2016, he and his group moved to USST. His scientific interests focus on quantum effects in biological systems, and bio-inspired materials.

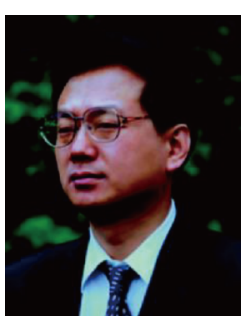

Lei Jiang is a professor at the TIPC, Chinese Academy of Sciences, and Beihang University. $\mathrm{He}$ is an academician of the Chinese Academy of Sciences, Academy of Sciences for the Developing World, and National Academy of Engineering, USA. He received his Bachelor's and Master's degrees from Jilin University, and $\mathrm{PhD}$ from the University of Tokyo. He worked as a post-doctoral fellow with Prof. Akira Fujishima and then as a senior researcher in the Kanagawa Academy of Sciences and Technology with Prof. Kazuhito Hashimoto. In 1999, he joined the Institute of Chemistry, Chinese Academy of Sciences as part of the Hundred Talents Program. In 2015, he and his group moved to TIPC. His scientific interests focus on bio-inspired, smart, multi-scale interfacial materials with superwettability. 


\section{离子通道宏观量子态: 神经信息载体}

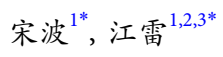

摘要 神经科学广泛发展了 70 多年, 然而对神经信息的本质仍缺乏 突破性认识. 最近报道, 离子通道量子相干态可能是神经信息的一 种新载体, 但是其物理表达式仍然未确定. 本文以 $K^{+}$通道为例, 从 理论上建立了神经离子通道量子态的概念模型, 展示了多离子通 道的宏观相干态. 该现象的物理机理是: (1) 单个离子通道中离子 振荡可以释放中红外光子, (2) 多个离子通道通过该光子可以实现 共振相干耦合. 环境场(如脑电波) 能够调节离子通道的相干性, 与 意识存在潜在的关联. 神经系统中还存在其他离子通道, 它们也可 形成各自的宏观相干态, 其机制与 $\mathrm{K}^{+}$通道相同. 环境场可以进一步 调控各种离子通道量子态, 使它们产生整体相干. 该现象类似于交 响乐: 不同的乐器(不同频率的离子通道及其相干态) 由乐队指挥 (环境场)统一调控, 实现音乐和声(整体宏观相干态). 这些研究发 现有望为神经科学提供新观点, 同时为神经系统相关疾病、健康 问题的治疗提供新策略. 